\section{Growing old gracefully}

\section{Linda Partridge}

Longevity, Senescence and the Genome. By Caleb E. Finch. University of Chicago Press: 1991. Pp. 922. \$49.95, $£ 39.95$.

THE word 'ageing' has different implications for wine and women. In Longevity, Senescence and the Genome Caleb Finch reacts to this diversity of meaning by banning the word from his book, preferring instead to confine himself to 'senescence'. As Finch points out, things can get better as well as worse during the course of life; very young animals are often more likely to die than those at the peak of their powers, and animals that grow as adults often become more reproductively successful as they do so. Finch has sown the seeds of some confusion by making the tacit assumption that similar mechanisms are responsible for these two kinds of change.

Although usages differ in important detail, gerontologists and evolutionary biologists both use the term 'ageing' in a general way to refer to the drop in biological performance late in life. Ever since the scientific study of ageing started with August Weismann, there has been a gulf between those working on the proximate mechanisms responsible and those interested in the ultimate evolutionary reasons for its existence. Finch makes a concerted effort to unite the two approaches, which is a thoroughly worthwhile undertaking, and he has drawn together a large and scattered literature on ageing, and looked for some generalizations. Few emerge, partly because the integration of gerontological and evolutionary approaches is incomplete.

Evolutionary biologists think they understand different kinds of age-related changes, at least in broad outline. Some events, such as those of embryonic development and the onset of reproduction, are the products of

\section{NEW JOURNALS ISSUE}

This year, Nature's annual new journals review supplement will appear in the issue of 3 October. Publishers and learned societies are invited to submit journals for review, taking note of the following criteria: - Journals that first appeared after June 1989 and issued at least four separate numbers by the end of April 1991 will be considered. - The deadline for submission is end of May. - Journals covering any aspect of science are eligible, but those dealing with clinical medicine, engineering and pure mathematics are excluded, as are publications of abstracts. -The journals must be published at least three times a year. $\bullet$ The main language used must be English.

When submitting journals for review, please send at least four different issues (the first, the most recent and any two others). natural selection, and are controlled by mechanisms ensuring that they occur at appropriate times in the life history. Senescence is not like development: there is no predictable set of events that occurs at similar times in all members of a species. Rather, almost anything that can go wrong does go wrong in at least some cases, and the timing and extent of decline is enormously variable. The haphazard nature of senescence can be explained if it is not the product of natural selection, and is in fact maladaptive. It can none the less evolve for two reasons. First, natural selection may fail to eliminate mutations that impair survival or reproduction in old age, because most of the carriers will have died of something else by then anyway. Second, there may be bad, but weakly selected, side-effects late in life of mutations that are advantageous earlier on. Either way, ageing is expected to evolve and to decrease the likelihood of both survival and reproduction later in life.

The rate of ageing that evolves will depend upon demography in nature. A risky way of life will produce high, externally imposed, age-independent mortality rates, and will lead to the evolution of rapid senescence. One of the unique strengths of the book is that changes in the rate of ageing are clearly distinguished from changes in death rate unrelated to age, and the former do indeed vary enormously between species: Finch quotes mortality rate doubling times of 27 years for river perch, eight years for elephants, six months for white-footed mice and one week for Drosophila melanogaster. $\mathrm{He}$ does not examine the association between hazard and the rate of senescence, which would have been interesting.

Against this theoretical background, some of the questions Finch poses do not make a lot of sense. For instance, he frequently refers to the idea that senescence is controlled by one or more pacemakers, which might reside in the genome. He therefore presents a review of the ways in which gene expression and fidelity of DNA replication change during the lifespan of well-studied organisms. This information is comprehensive, well presented and very interesting. But the attempt to find the top of a hierarchy of controls for ageing is likely to prove profitless if many processes go wrong simultaneously, as evolutionary theories predict.

We need to understand the details of what goes wrong during ageing if we are to stand any chance of informed medical intervention. This kind of approach has occupied countless gerontologists and has produced an almost unmanageably large amount of information. One prediction of evolutionary theories of ageing is that it is likely to be affected by many genes and to involve simultaneous decline in those processes responsible for continuing survival and fertility. As the data marshalled by Finch clearly demonstrate, the overall picture is confusing. Elephants loose their teeth whereas male marsupial mice Antechinus die from the side effects of the hormones responsible for suicidal rates of reproduction. The evolutionary approach would have something to offer in explaining this diversity. Most mechanistic theories of ageing are theories of damage through release of free radicals, somatic mutation, errors in protein synthesis, injury to joints and so on. Which of these particular hazards is most pressing will depend upon the way of life of the organism concerned. It must be presumed that when rates of ageing evolve, they do so through alteration in the extent to which at least some of these forms of damage are combatted. The interesting questions then become comparative: do bats repair their DNA more than small rodents and do storm petrels mop up free radicals more effectively than starlings? These are, of course, difficult questions to answer.

Finch takes a slightly different tack, and attempts to test the evolutionary theories by a detailed examination of what goes wrong in particular systems. The data are again interesting, but not obviously relevant. For instance, the fact that the ageing pigments lipofuscins accumulate at different rates in various cell types in mice does not seem to contradict the theory, as Finch suggests. What matters is how these different rates of change affect survival and reproduction. When Henry Ford inspected the scrap heaps to find out what economies in manufacture could be made, he was not interested in the numbers of scratches on the steering wheels and the cylinder heads.

An oddity of the text, although one with a distinguished pedigree in gerontology, is the lack of emphasis on the decline in fertility later in life. From the evolutionary point of view of genetic contribution to the next generation, this is as serious as the decline in survival, and the mechanisms producing it are of as much interest as those impairing survival. Finch claims that reproductive determinism, in other words a fixed quota of germ cells, is the only mechanism that would produce a decline in fertility with age. This seems an unlikely explanation in many cases, because individuals that are prevented from using germ cells early in life still show the later decline. One interesting point to emerge from the discussions of reproduction is that semelparity (breeding only once) does seem to be an evolutionary black hole from which repeated breeding does not re-evolve.

An enormous virtue of Finch's text is that it does not confine itself to the traditional model organisms for the study of ageing: the fruitfly, the nematode and the mouse. This is a treasure house of fascinating information about other organisms, which will undoubtedly pave the way for comparative work.

Linda Partridge is at the Institute of Cell, Animal and Population Biology, University of Edinburgh, West Main's Road, Edinburgh EH9 3JT, UK. 